\title{
Probabilistic Analysis of Power Assignments
}

\author{
Maurits de Graaf,, ${ }^{1,2}$ Bodo Manthey ${ }^{1}$ \\ ${ }^{1}$ Department of Applied Mathematics, University of Twente, Enschede, Netherlands; \\ e-mail:m.degraaf@utwente.nl; b.manthey@utwente.nl \\ ${ }^{2}$ Thales Nederland B. V., Huizen, Netherlands
}

Received 11 September 2015; accepted 16 September 2016

Published online 28 March 2017 in Wiley Online Library (wileyonlinelibrary.com).

DOI 10.1002/rsa.20714

\begin{abstract}
A fundamental problem for wireless ad hoc networks is the assignment of suitable transmission powers to the wireless devices such that the resulting communication graph is connected. The goal is to minimize the total transmit power in order to maximize the life-time of the network. Our aim is a probabilistic analysis of this power assignment problem. We prove complete convergence for arbitrary combinations of the dimension $d$ and the distance-power gradient $p$. Furthermore, we prove that the expected approximation ratio of the simple spanning tree heuristic is strictly less than its worst-case ratio of 2 .

Our main technical novelties are two-fold: First, we find a way to deal with the unbounded degree that the communication network induced by the optimal power assignment can have. Minimum spanning trees and traveling salesman tours, for which strong concentration results are known in Euclidean space, have bounded degree, which is heavily exploited in their analysis. Second, we apply a recent generalization of Azuma-Hoeffding's inequality to prove complete convergence for the case $p \geq d$ for both power assignments and minimum spanning trees (MSTs). As far as we are aware, complete convergence for $p>d$ has not been proved yet for any Euclidean functional. (C) 2017 The Authors Random Structures \& Algorithms Published by Wiley Periodicals, Inc. Random Struct. Alg., 51, 483-505, 2017
\end{abstract}

Keywords: Euclidean optimization problems, power assignments, average-case analysis, complete convergence

\section{INTRODUCTION}

Wireless ad hoc networks have received significant attention due to their many applications in, for instance, environmental monitoring or emergency disaster relief, where wiring

Correspondence to: Bodo Manthey

A preliminary version of this paper has been presented at the 39th Int. Symposium on Mathematical Foundations of Computer Science (MFCS 2014).

This is an open access article under the terms of the Creative Commons Attribution License, which permits use, distribution and reproduction in any medium, provided the original work is properly cited.

(C) 2017 The Authors Random Structures \& Algorithms Published by Wiley Periodicals, Inc. 
is difficult. Unlike most wired networks, wireless ad hoc networks lack a backbone infrastructure. Communication takes place either through single-hop transmission or by relaying through intermediate nodes. We consider the case of ad hoc wireless networks where each node can adjust its transmit power for the purpose of power conservation. In the assignment of transmit powers, two conflicting effects have to be taken into account: if the transmit powers are too low, the resulting network may be disconnected. If the transmit powers are too high, the nodes run out of energy quickly. The goal of the power assignment problem is to assign transmit powers to the transceivers such that the resulting network is connected and the sum of transmit powers is minimized [15].

\subsection{Problem Statement and Previous Results}

We consider a set of vertices $X \subseteq[0,1]^{d}$, which represent the sensors, $|X|=n$, and assume that $\|u-v\|^{p}$, for some $p \in \mathbb{R}$ (called the distance-power gradient or path loss exponent), is the power required to successfully transmit a signal from $u$ to $v$. This is called the powerattenuation model, where the strength of the signal decreases with $1 / r^{p}$ for distance $r$, and is a simple yet very common model for power assignments in wireless networks [21]. In practice, we typically have $1 \leq p \leq 6$ [18].

A power assignment pa : $X \rightarrow[0, \infty)$ is an assignment of transmit powers to the nodes in $X$. Given pa, we have an edge between two nodes $u$ and $v$ if both pa $(x), \operatorname{pa}(y) \geq\|x-y\|^{p}$. If the resulting graph is connected, we call it a $P A$ graph. Our goal is to find a PA graph and a corresponding power assignment pa that minimizes $\sum_{v \in X} \mathrm{pa}(v)$. Note that any PA graph $G=(X, E)$ induces a power assignment by $\operatorname{pa}(v)=\max _{u \in X:\{u, v\} \in E}\|u-v\|^{p}$.

PA graphs can in many aspects be regarded as a tree as we are only interested in connectedness, but it can contain more edges in general. However, we can simply ignore redundant edges and restrict ourselves to a spanning tree of the PA graph.

The minimal connected power assignment problem is NP-hard for $d \geq 2$ and APXhard for $d \geq 3$ [4]. For $d=1$, i.e., when the sensors are located on a line, the problem can be solved by dynamic programming [13]. A simple approximation algorithm for minimum power assignments is the minimum spanning tree heuristic (MST heuristic), which achieves a tight worst-case approximation ratio of 2 [13]. This has been improved by Althaus, Calinescu, Mandoiu, Prasad, Tchernvenski, and Zelinovsky [1], who devised an approximation algorithm that achieves an approximation ratio of 5/3. Despite the higher worst-case approximation ratio of the MST heuristic in relation to the algorithm of Althaus et al., analysis remains of interest due to the inherent simplicity of the algorithm. A first averagecase analysis of the MST heuristic was presented by de Graaf, Boucherie, Hurink, and van Ommeren [5]: First, they analyzed the expected approximation ratio of the MST heuristic for the (non-geometric, non-metric) case of independent edge lengths. Second, they proved convergence of the total power consumption of the assignment computed by the MST heuristic for the special case of $p=d$. They did not analyze the optimal power assignment. They left as open problems, first, an average-case analysis of the MST heuristic for random geometric instances and, second, the convergence of the value of the optimal power assignment.

\subsection{Our Contribution}

In this paper, we conduct an average-case analysis of the optimal power assignment problem for Euclidean instances. The points are drawn independently and uniformly from the $d$ dimensional unit hypercube $[0,1]^{d}$. We believe that probabilistic analysis is better-suited 
for performance evaluation in wireless ad hoc networks than worst-case analysis, as the positions of the sensors - in particular if deployed in areas that are difficult to access - are subjected to randomness.

Roughly speaking, our contributions are as follows:

1. We show that the power assignment functional has sufficiently nice properties in order to apply Yukich's general framework for Euclidean functionals [27] to obtain concentration results (Section 3).

2. Combining these insights with a recent generalization of the Azuma-Hoeffding bound by Warnke [26], we obtain concentration of measure and complete convergence of the power assignment functional for all combinations of $d$ and $p \geq 1$, even for the case $p \geq$ $d$ (Section 4). In addition, we obtain complete convergence for $p \geq d$ for minimumweight spanning tree functional. As far as we are aware, complete convergence for $p \geq d$ has not been proved yet for such functionals. The only exception we are aware of are minimum spanning trees for the special case $p=d$ [27, Sect. 6.4].

3. We provide a probabilistic analysis of the MST heuristic for the geometric case. We show that its expected approximation ratio is strictly smaller than its worst-case approximation ratio of 2 [13] for any $d$ and $p$ (Section 5).

Our main technical contributions are two-fold: First, we introduce a transmit power redistribution argument to deal with the unbounded degree that graphs induced by the optimal transmit power assignment can have. The unboundedness of the degree makes the analysis of the power assignment functional PA challenging. The reason is that removing a vertex can cause the graph to fall into a large number of components and it might be costly to connect these components without the removed vertex. In contrast, the degree of any minimum spanning tree, for which strong concentration results are known in Euclidean space for $p \leq d$, is bounded for every fixed $d$, and this is heavily exploited in the analysis. (The concentration result by de Graaf et al. [5] for the power assignment obtained from the MST heuristic also exploits that MSTs have bounded degree.)

Second, we apply a recent generalization of Azuma-Hoeffding's inequality [26] to prove complete convergence for the case $p \geq d$ for both power assignments and minimum spanning trees. We introduce the notion of typically smooth Euclidean functionals, prove convergence of such functionals, and show that minimum spanning trees and power assignments are typically smooth. In this sense, our proof of complete convergence provides an alternative and generic way to prove complete convergence, whereas Yukich's proof for minimum spanning trees is tailored to the case $p=d$. In order to prove complete convergence with our approach, one only needs to prove convergence in mean, which is often much simpler than complete convergence, and typically smoothness. Thus, we provide a simple method to prove complete convergence of Euclidean functionals along the lines of Yukich's result that, in the presence of concentration of measure, convergence in mean implies complete convergence [27, Corollary 6.4].

\section{DEFINITIONS AND NOTATION}

Throughout the paper, $d$ (the dimension) and $p$ (the distance-power gradient) are fixed constants. For three points $x, y, v$, we by $\overline{x v}$ the line through $x$ and $v$, and we denote by $\angle(x, v, y)$ the angle between $\overline{x v}$ and $\overline{y v}$. 
A Euclidean functional is a function $\mathrm{F}^{p}$ for $p>0$ that maps finite sets of points from the unit hypercube $[0,1]^{d}$ to some non-negative real number and is translation invariant and homogeneous of order $p$ [27, page 18]. From now on, we omit the superscript $p$ of Euclidean functionals, as $p$ is always fixed and clear from the context.

$\mathrm{PA}_{B}$ is the canonical boundary functional of PA (we refer to Yukich [27] for boundary functionals of other optimization problems): given a hyperrectangle $R \subseteq \mathbb{R}^{d}$ with $X \subseteq R$, this means that a solution is an assignment $\mathrm{pa}(x)$ of power to the nodes $x \in X$ such that

- $x$ and $y$ are connected if $\mathrm{pa}(x), \operatorname{pa}(y) \geq\|x-y\|^{p}$,

- $x$ is connected to the boundary of $R$ if the distance of $x$ to the boundary of $R$ is at most $\mathrm{pa}(x)^{1 / p}$, and

- the resulting graph, called a boundary PA graph, is either connected or consists of connected components that are all connected to the boundary.

Then $\mathrm{PA}_{B}(X, R)$ is the minimum value for $\sum_{x \in X} \mathrm{pa}(x)$ that can be achieved by a boundary PA graph. Note that in the boundary functional, no power is assigned to the boundary. It is straight-forward to see that $\mathrm{PA}$ and $\mathrm{PA}_{B}$ are Euclidean functionals for all $p>0$ according to Yukich [27, page 18].

For a hyperrectangle $R \subseteq \mathbb{R}^{d}$, let $\operatorname{diam} R=\max _{x, y \in R}\|x-y\|$ denote the diameter of $R$. For a Euclidean functional $\mathrm{F}$, let $\mathrm{F}(n)=\mathrm{F}\left(\left\{U_{1}, \ldots, U_{n}\right\}\right)$, where $U_{1}, \ldots, U_{n}$ are drawn uniformly and independently from $[0,1]^{d}$. Let

$$
\gamma_{\mathrm{F}}^{d, p}=\lim _{n \rightarrow \infty} \frac{\mathbb{E}(\mathrm{F}(n))}{n^{\frac{d-p}{d}}} .
$$

(In principle, $\gamma_{\mathrm{F}}^{d, p}$ need not exist, but it does exist for all functionals considered in this paper.)

A sequence $\left(R_{n}\right)_{n \in \mathbb{N}}$ of random variables converges in mean to a constant $\gamma$ if

$$
\lim _{n \rightarrow \infty} \mathbb{E}\left(\left|R_{n}-\gamma\right|\right)=0
$$

The sequence $\left(R_{n}\right)_{n \in \mathbb{N}}$ converges completely to a constant $\gamma$ if we have

$$
\sum_{n=1}^{\infty} \mathbb{P}\left(\left|R_{n}-\gamma\right|>\varepsilon\right)<\infty
$$

for all $\varepsilon>0$ [27, page 33].

Besides PA, we consider two other Euclidean functions: $\operatorname{MST}(X)$ denotes the length of the minimum spanning tree with lengths raised to the power $p$. PT $(X)$ denotes the total power consumption of the assignment obtained from the MST heuristic, again with lengths raised to the power $p$. The MST heuristic proceeds as follows: First, we compute a minimum spanning tree of $X$. Then let

$$
\operatorname{pa}(x)=\max \left\{\|x-y\|^{p} \mid\{x, y\} \text { is an edge of the MST }\right\} .
$$

By construction and a simple analysis, we have $\mathrm{MST}(X) \leq \mathrm{PA}(X) \leq \mathrm{PT}(X) \leq 2$. $\operatorname{MST}(X)[13]$.

For $n \in \mathbb{N}$, let $[n]=\{1, \ldots, n\}$. 


\section{PROPERTIES OF THE POWER ASSIGNMENT FUNCTIONAL}

After showing that optimal PA graphs can have unbounded degree and providing a lemma that helps solving this problem, we show that the power assignment functional fits into Yukich's framework for Euclidean functionals [27].

\subsection{Degrees and Cones}

As opposed to minimum spanning trees, whose maximum degree is bounded from above by a constant that depends only on the dimension $d$, a technical challenge is that the maximum degree in an optimal PA graph cannot be bounded by a constant in the dimension. This holds even for the simplest case of $d=1$ and $p>1$. We conjecture that the same holds also for $p=1$, but proving this seems to be more difficult and not to add much.

Lemma 3.1. For all $p>1$, all integers $d \geq 1$, and for infinitely many $n$, there exist instances of $n$ points in $[0,1]^{d}$ such that the unique optimal PA graph is a tree with a maximum degree of $n-1$.

Proof. Let $n$ be odd, and let $2 m+1=n$. Consider the instance

$$
X_{m}=\left\{a_{-m}, a_{-m+1}, \ldots, a_{0}, \ldots, a_{m-1}, a_{m}\right\}
$$

that consists of $m$ positive integers $a_{1}, \ldots, a_{m}, m$ negative integers $a_{-i}=-a_{i}$ for $1 \leq i \leq m$, and $a_{0}=0$. We assume that $a_{i+1} \gg a_{i}$ for all $i$. By scaling and shifting, we can achieve that $X$ fits into the unit interval.

A possible solution pa : $X_{m} \rightarrow \mathbb{R}^{+}$is assigning power $a_{i}^{p}$ to $a_{i}$ and $a_{-i}$ for $1 \leq i \leq m$ and power $a_{m}^{p}$ to 0 . In this way, all points are connected to 0 . We claim that this power assignment is the unique optimum. As $a_{m}=-a_{-m} \gg\left|a_{i}\right|$ for $|i|<m$, the dominant term in the power consumption $\Psi_{m}$ is $3 a_{m}^{p}$ (the power of $a_{m}, a_{-m}$, and $a_{0}=0$ ). Note that no other term in the total power consumption involves $a_{m}$.

We show that $a_{m}$ and $a_{-m}$ must be connected to 0 in an optimal PA graph. First, assume that $a_{m}$ and $a_{-m}$ are connected to different vertices. Then the total power consumption increases to about $4 a_{m}^{p}$ because $a_{ \pm m}$ is very large compared to $a_{i}$ for all $|i|<m$ (we say that $a_{m}$ is dominant). Second, assume that $a_{m}$ and $a_{-m}$ are connected to $a_{i}$ with $i \neq 0$. Without loss of generality, we assume that $i>0$ and, thus, $a_{i}>0$. Then the total power consumption is at least $2 \cdot\left(a_{m}+a_{i}\right)^{p}+\left(a_{m}-a_{i}\right)^{p} \geq 3 a_{m}^{p}+2 a_{m}^{p-1} a_{i}$. Because $a_{m}$ is dominant, this is strictly more than $\Psi_{m}$ because it contains the term $2 a_{m}^{p-1} a_{i}$, which contains the very large $a_{m}$ because $p>1$.

From now on, we can assume that $0=a_{0}$ is connected to $a_{ \pm m}$. Assume that there is some point $a_{i}$ that is connected to some $a_{j}$ with $i, j \neq 0$. Assume without loss of generality that $i>0$ and $|i| \geq|j|$. Assume further that $i$ is maximal in the sense that there is no $|k|>i$ such that $a_{k}$ is connected to some vertex other than 0 . We set $a_{i}$ 's power to $a_{i}^{p}$ and $a_{j}$ 's power to $\left|a_{j}\right|^{p}$. Then both are connected to 0 as 0 has already sufficient power to reach both. Furthermore, the PA graph is still connected: All vertices $a_{k}$ with $|k|>i$ are connected to 0 by the choice of $i$. If some $a_{k}$ with $|k| \leq i$ and $k \neq i, j$ was connected to $a_{i}$ before, then it has also sufficient power to reach 0 .

The power balance remains to be considered: If $j=-i$, then the energy of both $a_{i}$ and $a_{j}$ has been strictly decreased. Otherwise, $|j|<i$. The power of $a_{i}$ was at least $\left(a_{i}-a_{j}\right)^{p}$ 


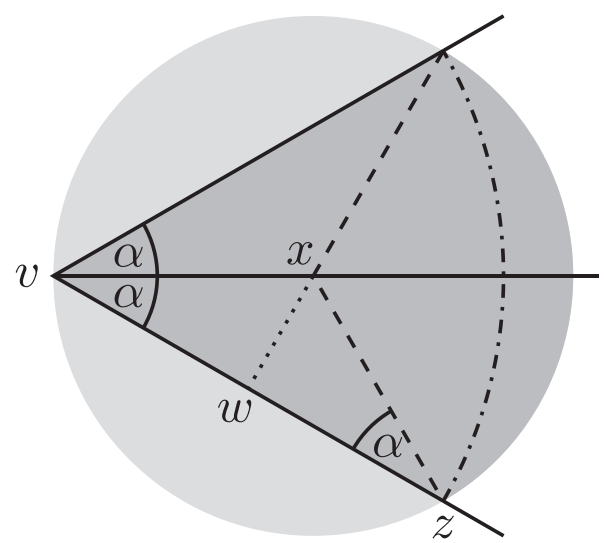

Fig. 1. Point $x$ can reach all points in the gray area because it can reach $v$. In particular, $x$ can reach all points that are no further away from $v$ than $z$. This includes all points to the left of the dash-dotted line. The dash-dotted line consists of points at a distance of $2 \cos (\alpha) \cdot\|x-v\|$ of $v$. A sufficient condition that the point $x$ can reach another point $y$ is that $y$ is contained in the dark-gray cone and is no further away from $v$ than indicated by the dash-dotted line.

before and is now $a_{i}^{p}$. The power of $a_{j}$ was at least $\left(a_{i}-a_{j}\right)^{p}$ before and is now $a_{j}^{p}$. Since $a_{i}$ dominates all $a_{j}$ for $|j|<i$, this decreases the power.

The unboundedness of the degree of PA graphs make the analysis of the functional PA challenging. The technical reason is that removing a vertex can cause the PA graph to fall into a non-constant number of components. The following lemma is the crucial ingredient to get over this "degree hurdle".

Lemma 3.2. Let $x, y \in X$, let $v \in[0,1]^{d}$, and assume that $x$ and $y$ have power $\mathrm{pa}(x) \geq$ $\|x-v\|^{p}$ and $\mathrm{pa}(y) \geq\|y-v\|^{p}$, respectively. Assume further that $\|x-v\| \leq\|y-v\|$ and that $\angle(x, v, y) \leq \alpha$ with $\alpha \leq \pi / 3$. Then the following holds:

(a) $\mathrm{pa}(y) \geq\|x-y\|^{p}$, i.e., $y$ has sufficient power to reach $x$.

(b) If $x$ and $y$ are not connected (i.e., $\left.\operatorname{pa}(x)<\|x-y\|^{p}\right)$, then $\|y-v\|>2 \cos (\alpha) \cdot\|x-v\|$.

Proof. Because $\alpha \leq \pi / 3$, we have $\|y-v\| \geq\|y-x\|$. This implies (a).

The point $x$ has sufficient power to reach any point within a radius of $\|x-v\|$ of itself. By (a), point $y$ has sufficient power to reach $x$. Thus, if $y$ is within a distance of $\|x-v\|$ of $x$, then also $x$ can reach $y$ and, thus, $x$ and $y$ are connected. We project $x, y$, and $v$ into the two-dimensional subspace spanned by the vectors $x-v$ and $y-v$. This yields a situation as depicted in Fig. 1. Since $\mathrm{pa}(x) \geq\|x-v\|^{p}$, point $x$ can reach all points in the light-gray region, thus in particular to all dark-gray points in the cone rooted at $v$. In particular, $x$ can reach all points that are no further away from $v$ than the point $z$. The triangle $v x z$ is isosceles. We split it into two triangles $v w x$ and $z x w$. This yields $\|z-v\|=2 \cos (\alpha)\|x-v\|$, which completes the proof of (b).

For instance, $\alpha=\pi / 6$ results in a factor of $\sqrt{3}=2 \cos (\pi / 3)$. In the following, we invoke this lemma always with $\alpha=\pi / 6$, but this choice is arbitrary as long as $\alpha<\pi / 3$, which causes $2 \cos (\alpha)$ to be strictly larger than 1 . 


\subsection{Deterministic Properties}

In this section, we state properties of the power assignment functional. Subadditivity (Lemma 3.3), superadditivity (Lemma 3.4), and growth bound (Lemma 3.5) are straightforward.

Lemma 3.3 (subadditivity). PA is subadditive [27, (2.2)] for all $p>0$ and all $d \geq 1$, i.e., for any point sets $X$ and $Y$ and any hyperrectangle $R \subseteq \mathbb{R}^{d}$ with $X, Y \subseteq R$, we have

$$
\mathrm{PA}(X \cup Y) \leq \mathrm{PA}(X)+\mathrm{PA}(Y)+O\left((\operatorname{diam} R)^{p}\right) .
$$

Proof. Let $T_{X}$ and $T_{Y}$ be optimal PA graphs for $X$ and $Y$, respectively. We connect these graphs by an edge of length at most $\operatorname{diam} R$. This yields a solution for $X \cup Y$, i.e., a PA graph, and the additional costs are bounded from above by the length of this edge to the power $p$, which is bounded by $O\left((\operatorname{diam} R)^{p}\right)$.

Lemma 3.4 (superadditivity). $\quad \mathrm{PA}_{B}$ is superadditive for all $p \geq 1$ and $d \geq 1$ [27, (3.3)], i.e., for any $X$, hyperrectangle $R \subseteq \mathbb{R}^{d}$ with $X \subseteq R$ and partition of $R$ into hyperrectangles $R_{1}$ and $R_{2}$, we have

$$
\mathrm{PA}_{B}^{p}(X, R) \geq \mathrm{PA}_{B}^{p}\left(X \cap R_{1}, R_{1}\right)+\mathrm{PA}_{B}^{p}\left(X \cap R_{2}, R_{2}\right)
$$

Proof. Let $T$ be an optimal boundary PA graph for $(X, R)$. This graph restricted to $R_{1}$ and $R_{2}$ yields boundary graphs $T_{1}$ and $T_{2}$ for $\left(X \cap R_{1}, R_{1}\right)$ and $\left(X \cap R_{2}, R_{2}\right)$, respectively. The sum of the costs of $T_{1}$ and $T_{2}$ is upper bounded by the costs of $T$ because $p \geq 1$ (splitting an edge at the border between $R_{1}$ and $R_{2}$ results in two edges whose sum of lengths to the power $p$ is at most the length of the original edge to the power $p$ ).

Lemma 3.5 (growth bound). For any $X \subseteq[0,1]^{d}$ and $p>0$ and $d \geq 1$, we have

$$
\mathrm{PA}_{B}(X) \leq \mathrm{PA}(X) \leq O\left(\max \left\{n^{\frac{d-p}{d}}, 1\right\}\right) .
$$

Proof. This follows from the growth bound for the MST [27, (3.7)], because MST $(X) \leq$ $\mathrm{PA}(X) \leq 2 \operatorname{MST}(X)$ for all $X[13]$. The inequality $\mathrm{PA}_{B}(X) \leq \mathrm{PA}(X)$ holds obviously.

The following lemma shows that PA is smooth, which roughly means that adding or removing a few points does not have a huge impact on the function value. Its proof requires Lemma 3.2 to deal with the fact that optimal PA graphs can have unbounded degree.

Lemma 3.6. The power assignment functional PA is smooth for all $0<p \leq d[27,(3.8)]$, i.e.,

$$
|\mathrm{PA}(X \cup Y)-\mathrm{PA}(X)|=O\left(|Y|^{\frac{d-p}{d}}\right)
$$

for all point sets $X, Y \subseteq[0,1]^{d}$.

Proof. One direction is straightforward: $\mathrm{PA}(X \cup Y)-\mathrm{PA}(X)$ is bounded by $\Psi=$ $O\left(|Y|^{\frac{d-p}{d}}\right)$, because the optimal PA graph for $Y$ has a value of at most $\Psi$ by Lemma 3.5. Then we can take the PA graph for $Y$ and connect it to the tree for $X$ with a single edge, which costs at most $O(1) \leq \Psi$ because $p \leq d$. 
For the other direction, consider the optimal PA graph $T$ for $X \cup Y$. The problem is that the degrees $\operatorname{deg}_{T}(v)$ of vertices $v \in Y$ can be unbounded (Lemma 3.1). (If the maximum degree were bounded, then we could argue in the same way as for the MST functional.) The idea is to exploit the fact that removing $v \in Y$ also frees some power. Roughly speaking, we proceed as follows: Let $v \in Y$ be a vertex of possibly large degree. We add the power of $v$ to some vertices close to $v$. The graph obtained from removing $v$ and distributing its energy has only a constant number of components.

To prove this, Lemma 3.2 is crucial. We consider cones rooted at $v$ with the following properties:

- The cones have a small angle $\alpha$, meaning that for every cone $C$ and every $x, y \in C$, we have $\angle(x, v, y) \leq \alpha$. We choose $\alpha=\pi / 6$.

- Every point in $[0,1]^{d}$ is covered by some cone.

- There is a finite number of cones. (This can be achieved because $d$ is a constant.)

Let $C_{1}, \ldots, C_{m}$ be these cones. By abusing notation, let $C_{i}$ also denote all points $x \in$ $C_{i} \cap(X \cup Y \backslash\{v\})$ that are adjacent to $v$ in $T$. For $C_{i}$, let $x_{i}$ be the point in $C_{i}$ that is closest to $v$ and adjacent to $v$ (breaking ties arbitrarily), and let $y_{i}$ be the point in $C_{i}$ that is farthest from $v$ and adjacent to $v$ (again breaking ties arbitrarily). (For completeness, we remark that $C_{i}$ can be ignored if $C_{i} \cap X=\emptyset$.) Let $\ell_{i}=\left\|y_{i}-v\right\|$ be the maximum distance of any point in $C_{i}$ to $v$, and let $\ell=\max _{i} \ell_{i}$.

We increase the power of $x_{i}$ by $\ell^{p} / m$. Since the power of $v$ is at least $\ell^{p}$ and we have $m$ cones, we can account for this with $v$ 's power because we remove $v$. Because $\alpha=\pi / 6$ and $x_{i}$ is closest to $v$, any point in $C_{i}$ is closer to $x_{i}$ than to $v$. According to Lemma 3.2(a), every point in $C_{i}$ has sufficient power to reach $x_{i}$. Thus, if $x_{i}$ can reach a point $z \in C_{i}$, then there is an established connection between them.

From this and increasing $x_{i}$ 's power to at least $\ell^{p} / m$, there is an edge between $x_{i}$ and every point $z \in C_{i}$ that has a distance of at most $\ell / \sqrt[p]{m}$ from $v$. We recall that $m$ and $p$ are constants.

Now let $z_{1}, \ldots, z_{k} \in C_{i}$ be the vertices in $C_{i}$ that are not connected to $x_{i}$ because $x_{i}$ has too little power. We assume that they are sorted by increasing distance from $v$. Thus, $z_{k}=y_{i}$. We can assume that no two $z_{j}$ and $z_{j^{\prime}}$ are in the same component after removal of $v$. Otherwise, we can simply ignore one of the edges $\left\{v, z_{j}\right\}$ and $\left\{v, z_{j^{\prime}}\right\}$ without changing the components.

Since $z_{j}$ and $z_{j+1}$ were connected to $v$ and they are not connected to each other, we can apply Lemma 3.2(b), which implies that $\left\|z_{j+1}-v\right\| \geq \sqrt{3} \cdot\left\|z_{j}-v\right\|$. Furthermore, $\left\|z_{1}-v\right\| \geq$ $\ell / \sqrt[p]{m}$ by assumption. Iterating this argument yields $\ell=\left\|z_{k}-v\right\| \geq \sqrt{3}^{k-1}\left\|z_{1}-v\right\| \geq$ $\sqrt{3}^{k-1} \cdot \ell / p \sqrt{m}$. This implies $k \leq \log _{\sqrt{3}}(\sqrt[p]{m})+1$. Thus, removing $v$ and redistributing its energy as described causes the PA graph to fall into at most a constant number of components. Removing $|Y|$ points causes the PA graph to fall into at most $O(|Y|)$ components. These components can be connected with costs $O\left(|Y|^{\frac{d-p}{d}}\right)$ by choosing one point per component and applying Lemma 3.5.

Lemma 3.7. $\mathrm{PA}_{B}$ is smooth for all $1 \leq p \leq d[27,(3.8)]$.

Proof. The idea is essentially identical to the proof of Lemma 3.6, and we use the same notation. Again, one direction is easy. For the other direction, note that every vertex of $G=(X, E)$, with $E$ induced by pa is connected to at most one point at the boundary. We use 
the same kind of cones as for Lemma 3.6. Let $v \in G$ be a vertex that we want to remove. We ignore $v$ 's possible connection to the boundary and proceed with the remaining connections. In this way, we obtain a forest with $O(|Y|)$ components. We compute a boundary PA graph for one vertex of each component and are done because of Lemma 3.5 and in the same way as in the proof of Lemma 3.6.

Crucial for convergence of $\mathrm{PA}$ is that $\mathrm{PA}$, which is subadditive, and $\mathrm{PA}_{B}$, which is superadditive, are close to each other. Then $\mathrm{PA}$ and $\mathrm{PA}_{B}$ are approximately both subadditive and superadditive. The following lemma states that indeed PA and $\mathrm{PA}_{B}$ do not differ too much for $1 \leq p<d$.

Lemma 3.8. $\mathrm{PA}$ is point-wise close to $\mathrm{PA}_{B}$ for $1 \leq p<d$ [27, (3.10)], i.e.,

$$
\left|\mathrm{PA}^{p}(X)-\mathrm{PA}_{B}^{p}\left(X,[0,1]^{d}\right)\right|=o\left(n^{\frac{d-p}{d}}\right)
$$

for every set $X \subseteq[0,1]^{d}$ of $n$ points.

Proof. Let $T$ be an optimal boundary PA graph for $X$. Let $Q \subseteq X$ be the set of points that have a connection to the boundary in $T$ and let $\partial Q$ be the corresponding points on the boundary. If we remove the connections to the boundary, we obtain a graph $T^{\prime}$. We can assume that $Q$ contains exactly one point per connected component of the graph $T^{\prime}$.

We use the same dyadic decomposition as Yukich [27, proof of Lemma 3.8]. This yields that the sum of transmit powers used to connect to the boundary is bounded by the maximum of $O\left(n^{\frac{d-p-1}{d-1}}\right)$ and $O(\log n)$ for $p \leq d-1$ and by a constant for $p \in(d-1, d)$. We omit the proof as it is basically identical to Yukich's proof.

We compute a minimum-weight spanning tree $Z$ of $\partial Q$. (Note that we indeed compute an MST and not a PA. This is because the MST has bounded degree and PA and MST differ by at most a factor of 2.) This MST $Z$ has a weight of

$$
O\left(\max \left\{n^{\frac{d-1-p}{d-1}}, 1\right\}\right)=o\left(n^{\frac{d-p}{d}}\right)
$$

according to the growth bound for MST [27, (3.7)]. and because $d>p$. If two points $\tilde{q}, \tilde{q}^{\prime} \in \partial Q$ are connected by an edge in this tree, then we connect the corresponding points $q, q^{\prime} \in Q$.

The question that remains is by how much the power of the vertices in $Q$ has to be increased in order to allow the connections as described above. If $q, q^{\prime} \in Q$ are connected, then an upper bound for their power is given by the $p$-th power of their distances to the boundary points $\tilde{q}$ and $\tilde{q}^{\prime}$ plus the length of the edge connecting $\tilde{q}$ and $\tilde{q}^{\prime}$. Applying the triangle inequality for powers of metrics twice, the energy needed for connecting $q$ and $q^{\prime}$ is at most $4^{p}=O(1)$ times the sum of these distances. Since the degree of $Z$ is bounded, every vertex in $Q$ contributes to only a constant number of edges and, thus, only to the power consumption of a constant number of other vertices. Thus, the total additional power needed is bounded by a constant times the power of connecting $Q$ to the boundary plus the power to use $Z$ as a PA graph. Because of the triangle inequality for powers of metrics, the bounded degree of every vertex of $\partial Q$ in $Z$, and because of the dyadic decomposition mentioned above, the increase of power is in compliance with the statement of the lemma. 
Remark 3.9. Lemma 3.8 is an analogue of its counterpart for MST, TSP, and matching [ 27 , Lemma 3.7] in terms of the bounds. Namely, we obtain

$$
\left|\mathrm{PA}(X)-\mathrm{PA}_{B}(X)\right| \leq \begin{cases}O\left(|X|^{\frac{d-p-1}{d-1}}\right) & \text { if } 1 \leq p<d-1, \\ O(\log |X|) & \text { if } p=d-1 \neq 1, \\ O(1) & \text { if } d-1<p<d \text { or } p=d-1=1 .\end{cases}
$$

\subsection{Probabilistic Properties}

For $p>d$, smoothness is not guaranteed to hold, and for $p \geq d$, point-wise closeness is not guaranteed to hold. But similar properties typically hold for random point sets, namely smoothness in mean (Definition 3.14) and closeness in mean (Definition 3.16). In the following, let $X=\left\{U_{1}, \ldots, U_{n}\right\}$. Recall that $U_{1}, \ldots, U_{n}$ are drawn uniformly and independently from $[0,1]^{d}$.

Before proving smoothness in mean, we need a statement about the longest edge in an optimal PA graph and boundary PA graph. The bound is asymptotically equal to the bound for the longest edge in an MST [7, 14, 19].

To prove our bound for the longest edge in optimal PA graphs (Lemma 3.12), we need the following two lemmas. Lemma 3.10 is essentially equivalent to a result by Kozma, Lotker, and Stupp [14], but they do not state the probability explicitly. Lemma 3.11 is a straightforward consequence of Lemma 3.10. Variants of both lemmas are known [7, 19, 20, 25], but, for completeness, we state and prove both lemmas in the forms that we need.

Lemma 3.10. For every $\beta>0$, there exists $a c_{\text {ball }}=c_{\text {ball }}(\beta, d)$ such that, with a probability of at least $1-n^{-\beta}$, every hyperball of radius $r_{\text {ball }}=c_{\text {ball }} \cdot(\log n / n)^{1 / d}$ and with center in $[0,1]^{d}$ contains at least one point of $X$ in its interior.

Proof. We sketch the simple proof. Fix $\beta>0$ arbitrarily. We cover $[0,1]^{d}$ with hypercubes of side length $\Omega\left(r_{\text {ball }}\right)$ such that every hyperball of radius $r_{\text {ball }}-$ even if its center is in a corner (for a point on the boundary, still at least a $2^{-d}=\Theta(1)$ fraction is within $[0,1]^{d}$ ) - fully contains at least one box. The probability that such a box does not contain a point, which is necessary for a ball to be empty, is at most $\left(1-\Omega\left(r_{\text {ball }}\right)^{d}\right)^{n} \leq n^{-\Omega(1)}$ by independence of the points in $X$ and the definition of $r_{\text {ball }}$. The rest of the proof follows by a union bound over all $O(n / \log n)$ boxes.

We also need the following lemma, which essentially states that if $z$ and $z^{\prime}$ are sufficiently far away, then there is - with high probability according to Lemma 3.10 - always a point $y$ between $z$ and $z^{\prime}$ in the following sense: the distance of $y$ to $z$ is within a predefined upper bound $2 r_{\text {ball }}$, and $y$ is closer to $z^{\prime}$ than $z$.

Lemma 3.11. Assume that every hyperball of radius $r_{\text {ball }}$ with center in $[0,1]^{d}$ contains at least one point of $X$. Then the following holds: For every choice of $z, z^{\prime} \in[0,1]^{d}$ with $\left\|z-z^{\prime}\right\| \geq 2 r_{\text {ball }}$, there exists a point $y \in X$ with the properties

- $\|z-y\| \leq 2 r_{\text {ball }}$ and

- $\left\|z^{\prime}-y\right\|<\left\|z^{\prime}-z\right\|$.

Proof. The set of candidates for $y$ contains a ball of radius $r_{\text {ball }}$, namely a ball of this radius whose center is at a distance of $r_{\text {ball }}$ from $z$ on the line between $z$ and $z^{\prime}$. 
Lemma 3.12 (longest edge). For every $\beta>0$, there exists a $c_{\text {edge }}=c_{\text {edge }}(\beta)$ such that, with a probability of at least $1-n^{-\beta}$, every edge of an optimal $P A$ graph and an optimal boundary PA graph $\mathrm{PA}_{B}$ is of length at most $r_{\text {edge }}=c_{\text {edge }} \cdot(\log n / n)^{1 / d}$.

Proof. We restrict ourselves to considering PA graphs. The proof for boundary PA graphs is almost identical.

Let $T$ be any PA graph. Let $c_{\text {edge }}=4 k^{1 / p} c_{\text {ball }} /\left(1-\sqrt{3}^{-p}\right)^{1 / p}$, where $k$ is an upper bound for the number of vertices without a pairwise connection at a distance between $r$ and $r / \sqrt{3}$ for arbitrary $r$. It follows from Lemma 3.2 and its proof that $k$ is a constant that depends only on $p$ and $d$.

Note that $c_{\text {edge }}>2 c_{\text {ball }}$. We are going to show that the following holds: Assume that every hyperball of radius $r_{\text {ball }}$ with center in $[0,1]^{d}$ contains at least one point (this is likely according to Lemma 3.10). Then for every PA graph $T$ that contains an edge that is longer than $r_{\text {edge }}$, we can find a better PA graph, which shows that $T$ is not optimal. Since the probability of the assumption is at least $1-n^{-\beta}$ by Lemma 3.10, the lemma is proved.

Now assume that every ball of radius $r_{\text {ball }}$ contains at least one point. This implies that the conclusion of Lemma 3.11 holds. Let $T$ be any PA graph that contains an edge of length at least $r_{\text {edge }}$. Let $v$ be a vertex incident to the longest edge of $T$, and let $r_{\text {big }}>r_{\text {edge }}$ be the length of a longest edge. (The longest edge is unique with a probability of 1 . The node $v$ is not unique as the longest edge connects two nodes.) We decrease the power of $v$ to $r_{\text {big }} / \sqrt{3}$. This implies that $v$ loses contact to some points - otherwise, the power assignment was clearly not optimal.

Let $x_{1}, \ldots, x_{k^{\prime}}$ with $k^{\prime} \leq k$ be the points that were connected to $v$ but are in different connected components than $v$ after decreasing $v$ 's power. This is because the only nodes that might lose their connection to $v$ are within a distance between $r_{\text {big }} / \sqrt{3}$ and $r_{\text {big }}$, and there are at most $k$ such nodes without a pairwise connection.

Consider $x_{1}$. Let $z_{0}=v$. According to Lemma 3.11, there is a point $z_{1}$ that is closer to $x_{1}$ and at most $2 r_{\text {ball }}$ away from $v$. Iteratively for $i=1,2, \ldots$, we distinguish three cases until this process stops:

(i) $z_{i}$ belongs to the same component as $v$. The process continues, and we can apply Lemma 3.11 to $z_{i}$ and $x_{1}$ and find a point $z_{i+1}$ that is closer to $x_{1}$ than $z_{i}$ and at most at a distance of $2 r_{\text {ball }}$ of $z_{i}$.

(ii) $z_{i}$ belongs to the same component as $x_{j}$ for some $j\left(z_{i}\right.$ is closer to $x_{1}$ than $z_{i-1}$, but this does not imply $j=1$ ). We increase $z_{i}$ 's power such that $z_{i}$ is able to reach $z_{i-1}$. If $i>1$, then we also increase $z_{i-1}$ 's power accordingly. This stops the process.

(iii) $z_{i}$ is within a distance of at most $2 r_{\text {ball }}$ of some $x_{j}$. In this case, we increase the energy of $z_{i}$ such that $z_{i}$ and $x_{j}$ are connected. (The energy of $x_{j}$ is sufficiently large anyhow.) This stops the process.

Running this process once decreases the number of connected components by one and costs at most $2\left(2 r_{\text {ball }}\right)^{p}=2^{p+1} r_{\text {ball }}^{p}$ additional power. We run this process $k^{\prime} \leq k$ times, thus spending at most $k 2^{p+1} r_{\text {ball }}^{p}$ of additional power. In this way, we obtain a valid PA graph.

We have to show that the new PA graph indeed saves power. By decreasing $v$ 's power, we save an amount of $r_{\text {big }}^{p}-\left(r_{\text {big }} / \sqrt{3}\right)^{p}>\left(1-\sqrt{3}^{-p}\right) \cdot r_{\text {edge }}^{p}$. By the choice of $c_{\text {edge }}$, the saved amount of energy exceeds the additional amount of $k 2^{p+1} r_{\text {ball }}^{p}$. This contradicts the optimality of the PA graph $T$ with the edge of length $r_{\text {big }}>r_{\text {edge }}$. 
Remark 3.13. Since the longest edge has length at most $r_{\text {edge }}$ with high probability, i.e., with a probability of $1-n^{-\Omega(1)}$, and any ball of radius $r_{\text {edge }}$ contains roughly $O(\log n)$ points due to Chernoff's bound [17, Chapter 4], the maximum degree of an optimum PA graph of a random point set is $O(\log n)$ with high probability - contrasting Lemma 3.1.

Yukich gave two different notions of smoothness in mean [27, (4.13) and (4.20) \& (4.21)]. We use the stronger notion, which implies the other.

Definition 3.14 (smooth in mean [27, (4.20), (4.21)]). A Euclidean functional $\mathrm{F}$ is called smooth in mean if, for every constant $\beta>0$, there exists a constant $c=c(\beta)$ such that the following holds with a probability of at least $1-n^{-\beta}$ :

$$
|\mathrm{F}(n)-\mathrm{F}(n \pm k)| \leq c k \cdot\left(\frac{\log n}{n}\right)^{p / d}
$$

and

$$
\left|\mathrm{F}_{B}(n)-\mathrm{F}_{B}(n \pm k)\right| \leq c k \cdot\left(\frac{\log n}{n}\right)^{p / d} .
$$

for all $0 \leq k \leq n / 2$.

Lemma 3.15. $\mathrm{PA}_{B}$ and $\mathrm{PA}$ are smooth in mean for all $p>0$ and all $d$.

Proof. The bound $\mathrm{PA}(n+k) \leq \mathrm{PA}(n)+O\left(k \cdot\left(\frac{\log n}{n}\right)^{\frac{p}{d}}\right)$ follows from the fact that for all $k$ additional vertices, with a probability of at least $1-n^{-\beta^{\prime}}$ for any $\beta^{\prime}>\beta>0$ (where $\beta$ is the constant in Definition 3.14), there is a vertex among the first $n$ within a distance of at most $O\left((\log n / n)^{1 / d}\right)$ according to Lemma $3.10\left(\beta^{\prime}\right.$ influences the constant hidden in the $O)$. Thus, we can connect any of the $k$ new vertices with costs of $O\left((\log n / n)^{p / d}\right)$ to the optimal PA graph for the $n$ nodes.

Let us now show the reverse inequality $\mathrm{PA}(n) \leq \mathrm{PA}(n+k)+O\left(k \cdot\left(\frac{\log n}{n}\right)^{\frac{p}{d}}\right)$. To do this, we show that with a probability of at least $1-n^{-\beta^{\prime}}$ (for some $\beta^{\prime}>\beta$ ), we have

$$
\mathrm{PA}(n) \leq \mathrm{PA}(n+1)+O\left(\left(\frac{\log n}{n}\right)^{\frac{p}{d}}\right) .
$$

Then we iterate $k$ times to obtain the bound we aim for.

The proof of (1) is similar to the analogous inequality in Yukich's proof [27, Lemma 4.8]. The only difference is that we first have to redistribute the power of the point $U_{n+1}$ to its closest neighbors as in the proof of Lemma 3.6. In this way, removing $U_{n+1}$ results in a constant number of connected components. The longest edge incident to $U_{n+1}$ has a length of $O\left((\log n / n)^{1 / d}\right)$ with a probability of at least $1-n^{-\beta^{\prime}}$. Thus, we can connect these constant number of components with extra power of at most $O\left((\log n / n)^{p / d}\right)$.

The proof of

$$
|\mathrm{PA}(n)-\mathrm{PA}(n-k)|=O\left(k \cdot\left(\frac{\log n}{n}\right)^{\frac{p}{d}}\right)
$$

and the statement

$$
\left|\mathrm{PA}_{B}(n)-\mathrm{PA}_{B}(n \pm k)\right|=O\left(k \cdot\left(\frac{\log n}{n}\right)^{\frac{p}{d}}\right)
$$

for the boundary functional are almost identical. 
Definition 3.16 (close in mean [27, (4.11)]). A Euclidean functional $\mathrm{F}$ is close in mean to its boundary functional $\mathrm{F}_{B}$ if

$$
\mathbb{E}\left(\left|\mathrm{F}(n)-\mathrm{F}_{B}(n)\right|\right)=o\left(n^{\frac{d-p}{d}}\right) .
$$

Lemma 3.17. $\mathrm{PA}$ is close in mean to $\mathrm{PA}_{B}$ for all $d$ and $p \geq 1$.

Proof. It is clear that $\mathrm{PA}_{B}(X) \leq \mathrm{PA}(X)$ for all $X$. Thus, in what follows, we prove that $\mathrm{PA}(X) \leq \mathrm{PA}_{B}(X)+o\left(n^{\frac{d-p}{d}}\right)$ holds with a probability of at least $1-n^{-\beta}$ for every $\beta>0$. This implies closeness in mean.

With a probability of at least $1-n^{-\beta^{\prime}}$ for some sufficiently large constant $\beta^{\prime}>\beta>0$, the longest edge in the graph that realizes $\mathrm{PA}_{B}(X)$ has a length of $c_{\text {edge }} \cdot(\log n / n)^{1 / d}$ with $c_{\text {edge }}=c_{\text {edge }}\left(\beta^{\prime}\right)$ (Lemma 3.12). Thus, with a probability of at least $1-n^{-\beta^{\prime}}$ for any constant $\beta^{\prime}>\beta>0$, only vertices within a distance of at most $c_{\text {edge }} \cdot(\log n / n)^{1 / d}$ of the boundary are connected to the boundary. As the $d$-dimensional unit cube is bounded by $2 d$ hyperplanes, the expected number of vertices that are so close to the boundary is bounded from above by $c_{\text {edge }} n 2 d \cdot(\log n / n)^{1 / d}=O\left((\log n)^{1 / d} n^{\frac{d-1}{d}}\right)$. With a probability of at least $1-n^{-\beta}$, this number is exceeded by no more than a constant factor because of Chernoff's bound.

By Remark 3.13, the maximum degree of any vertex is $O(\log n)$ with a probability of at least $1-n^{-\Omega(1)}$. Thus, removing the vertices close to the boundary as described above causes the boundary PA graph to fall apart into at most $O\left((\log n)^{1+\frac{1}{d}} \cdot n^{\frac{d-1}{d}}\right)$ components. We choose one vertex of every component and start the process described in the proof of Lemma 3.12 to connect all of them. The costs per connection is bounded from above by $O\left((\log n / n)^{p / d}\right)$ with a probability of $1-n^{-\beta}$ for any constant $\beta>0$. Thus, the total costs are bounded from above by

$$
O\left((\log n / n)^{p / d}\right) \cdot O\left((\log n)^{1+\frac{1}{d}} n^{\frac{d-1}{d}}\right)=O\left((\log n)^{\frac{d+p-1}{d}} \cdot n^{\frac{d-1-p}{d}}\right)=o\left(n^{\frac{d-p}{d}}\right)
$$

with a probability of at least $1-n^{-\beta}$ for any constant $\beta>0$.

\section{CONVERGENCE}

\subsection{Standard Convergence}

Our findings of Sections 3.2 yield complete convergence of PA for $p<d$ (Theorem 4.1). Together with the probabilistic properties of Section 3.3, we obtain convergence in mean in a straightforward way for all combinations of $d$ and $p$ (Theorem 4.2). In Sections 4.2 and 4.3, we prove complete convergence for $p \geq d$.

Theorem 4.1. For all $d$ and $p$ with $1 \leq p<d$, there exists a constant $\gamma_{\mathrm{PA}}^{d, p}$ such that

$$
\frac{\mathrm{PA}^{p}(n)}{n^{\frac{d-p}{d}}}
$$

converges completely to $\gamma_{\mathrm{PA}}^{d, p}$. 
Proof. This follows from the results in Section 3.2 together with results by Yukich [27, Theorem 4.1].

Theorem 4.2. For all $p \geq 1$ and $d \geq 1$, there exists a constant $\gamma_{\mathrm{PA}}^{d, p}$ (equal to the constant of Theorem 4.1 for $p<d$ ) such that

$$
\lim _{n \rightarrow \infty} \frac{\mathbb{E}\left(\mathrm{PA}^{p}(n)\right)}{n^{\frac{d-p}{d}}}=\lim _{n \rightarrow \infty} \frac{\mathbb{E}\left(\mathrm{PA}_{B}^{p}(n)\right)}{n^{\frac{d-p}{d}}}=\gamma_{\mathrm{PA}}^{d, p} .
$$

Proof. This follows from the results in Sections 3.2 and 3.3 together with results by Yukich [27, Theorem 4.5].

\subsection{Concentration with Warnke's Inequality}

McDiarmid's or Azuma-Hoeffding's inequality are powerful tools to prove concentration of measure for a function that depends on many independent random variables, all of which have only a bounded influence on the function value. If we consider smoothness in mean (see Lemma 3.15), then we have the situation that the influence of a single variable is typically very small (namely $\left.O\left((\log n / n)^{p / d}\right)\right)$, but can be quite large in the worst case (namely $O(1)$ ). Unfortunately, this situation is not covered by McDiarmid's or Azuma-Hoeffding's inequality. Fortunately, Warnke [26] proved a generalization specifically for the case that the influence of single variables is typically bounded and fulfills a weaker bound in the worst case.

The following theorem is a simplified version of a result by Lutz Warnke [26, Theorem 1.2, Remark 1].

Theorem 4.3 (Warnke [26, Theorem 1.2, Remark 1]). Let $\mathrm{F}:\left([0,1]^{d}\right)^{n} \rightarrow \mathbb{R}$. Suppose that there are numbers $c_{\mathrm{good}} \leq c_{\mathrm{bad}}$ and an event $\Gamma$ such that the function $\mathrm{F}:\left([0,1]^{d}\right)^{n} \rightarrow \mathbb{R}$ satisfies

$$
\begin{aligned}
\max _{i \in[n]} \max _{x_{1}, \ldots, x_{n}, x_{i}^{\prime} \in[0,1]^{d}}\left|\mathrm{~F}\left(x_{1}, \ldots, x_{n}\right)-\mathrm{F}\left(x_{1}, \ldots, x_{i-1}, x_{i}^{\prime}, x_{i+1}, \ldots, x_{n}\right)\right| \\
\leq \begin{cases}c_{\text {good }} & \text { if }\left(x_{1}, \ldots, x_{n}\right) \in \Gamma \text { and } \\
c_{\text {bad }} & \text { otherwise. }\end{cases}
\end{aligned}
$$

Then, for any $t \geq 0$ and $\gamma \in(0,1]$ and $\eta=c_{\mathrm{good}}+\gamma c_{\mathrm{bad}}$, we have

$$
\mathbb{P}(\mathrm{F}(n) \geq \mathbb{E}(\mathrm{F}(n))+t) \leq \exp \left(-\frac{t^{2}}{2 n \eta^{2}}\right)+\frac{n}{\gamma} \cdot \mathbb{P}(\neg \Gamma) .
$$

Next, we introduce typical smoothness, which means that, with high probability, a single point does not have a significant influence on the value of $F$, and we apply Theorem 4.3 for typically smooth functionals $\mathrm{F}$. The bound of $c \cdot(\log n / n)^{p / d}$ in Definition 4.4 below for the typical influence of a single point is somewhat arbitrary, but works for PA and MST. This bound is also essentially the smallest possible, as there can be regions of diameter $c^{\prime} \cdot(\log n / n)^{1 / d}$ for some small constant $c^{\prime}>0$ that contain no or only a single point. It might be possible to obtain convergence results for other functionals by using a larger $c_{\text {good }}$ in the following definition. 
Definition 4.4 (typically smooth). A Euclidean functional $\mathrm{F}$ is typically smooth if, for every $\beta>0$, there exists a constant $c=c(\beta)$ such that

$$
\max _{x \in[0,1]^{d}, i \in[n]}\left|\mathrm{F}\left(U_{1}, \ldots, U_{n}\right)-\mathrm{F}\left(U_{1}, \ldots, U_{i-1}, x, U_{i+1}, \ldots, U_{n}\right)\right| \leq c \cdot\left(\frac{\log n}{n}\right)^{p / d}=c_{\text {good }}
$$

with a probability of at least $1-n^{-\beta}$ and

$$
\max _{x_{1}, \ldots, x_{n} \in[0,1]^{d}, i \in[n], x_{i}^{\prime} \in[0,1]^{d}}\left|\mathrm{~F}\left(x_{1}, \ldots, x_{n}\right)-\mathrm{F}\left(x_{1}, \ldots, x_{i-1}, x_{i}^{\prime}, x_{i+1}, \ldots, x_{n}\right)\right|=O(1)=c_{\mathrm{bad}} .
$$

Theorem 4.5 (concentration of typically smooth functionals). Let $p, d \geq 1$. Assume that $\mathrm{F}$ is typically smooth. Then

$$
\mathbb{P}(|\mathrm{F}(n)-\mathbb{E}(\mathrm{F}(n))| \geq t) \leq O\left(n^{-\beta}\right)+\exp \left(-\frac{t^{2} n^{\frac{2 p}{d}-1}}{C(\log n)^{2 p / d}}\right)
$$

for an arbitrarily large constant $\beta>0$ and another constant $C>0$ that depends on $\beta$.

Proof. We use Theorem 4.3. The event $\Gamma$ is that any point can change the value only by at most $O\left((\log n / n)^{p / d}\right)$. Thus, $c_{\text {good }}=O\left((\log n / n)^{p / d}\right)$ and $c_{\text {bad }}=O(1)$. The probability that we do not have the event $\Gamma$ is bounded by $O\left(n^{-\beta}\right)$ for an arbitrarily large constant $\beta$ by typical smoothness. This only influences the constant $c$ in the definition of $c_{\text {good }}$ in Definition 4.4.

We choose $\gamma=\Theta\left((\log n / n)^{p / d}\right)$. In the notation of Theorem 4.3, we have $\eta=O(\gamma)$, which is possible as $c_{\text {bad }}-c_{\text {good }} \approx c_{\text {bad }}=\Theta(1)$. Using the conclusion of Theorem 4.3 yields

$$
\begin{aligned}
\mathbb{P}(|\mathrm{F}(n)-\mathbb{E}(\mathrm{F}(n))| \geq t) & \leq \frac{2 n}{\gamma} \cdot \mathbb{P}(\neg \Gamma)+\exp \left(-\frac{t^{2} n^{2 p / d}}{n C(\log n)^{2 p / d}}\right) \\
& \leq O\left(n^{-\beta}\right)+\exp \left(-\frac{t^{2} n^{2 p / d}}{n C(\log n)^{2 p / d}}\right)
\end{aligned}
$$

for some constant $C>0$. Here, $\beta$ can be chosen arbitrarily large.

The following corollary is an immediate consequence of the theorem above. It suffices to prove complete convergence of typically smooth Euclidean functionals.

Corollary 4.6. Let $p, d \geq 1$. Assume that $\mathrm{F}$ is typically smooth. Then

$$
\mathbb{P}\left(|\mathrm{F}(n)-\mathbb{E}(\mathrm{F}(n))|>C n^{\frac{1}{2}-\frac{p}{d}}(\log n)^{\frac{1}{2}+\frac{p}{d}}\right) \leq O\left(n^{-\beta}\right)
$$

for any constant $\beta$ and $C=C(\beta)$ depending on $\beta$.

Proof. This follows immediately from Theorem 4.5 by choosing $t=C n^{\frac{1}{2}-\frac{p}{d}}(\log n)^{\frac{1}{2}+\frac{p}{d}}$. 


\subsection{Complete Convergence for $p \geq d$}

In this section, we prove that typical smoothness (Definition 4.4) suffices for complete convergence. This implies complete convergence of MST and PA by Lemma 4.8 below.

Theorem 4.7. Let $p, d \geq 1$. Assume that $\mathrm{F}$ is typically smooth and $\mathrm{F}(n) / n^{\frac{d-p}{d}}$ converges in mean to $\gamma_{\mathrm{F}}^{d, p}$. Then $\mathrm{F}(n) / n^{\frac{d-p}{d}}$ converges completely to $\gamma_{\mathrm{F}}^{d, p}$.

Proof. Fix any $\varepsilon>0$. Since

$$
\lim _{n \rightarrow \infty} \mathbb{E}\left(\frac{\mathrm{F}(n)}{n^{\frac{d-p}{d}}}\right)=\gamma_{\mathrm{F}}^{d, p}
$$

there exists an $n_{0}$ such that

$$
\mathbb{E}\left(\frac{\mathrm{F}(n)}{n \frac{d-p}{d}}\right) \in\left[\gamma_{\mathrm{F}}^{d, p}-\frac{\varepsilon}{2}, \gamma_{\mathrm{F}}^{d, p}+\frac{\varepsilon}{2}\right]
$$

for all $n \geq n_{0}$.

Furthermore, there exists an $n_{1}$ such that, for all $n \geq n_{1}$, the probability that the random variable $\mathrm{F}(n) / n^{\frac{d-p}{d}}$ deviates by more than $\varepsilon / 2$ from its expected value is smaller than $n^{-2}$ for all $n \geq n_{1}$. To see this, we use Corollary 4.6 and observe that the right-hand side of (4) is $O\left(n^{-2}\right)$ for sufficiently large $\beta$ and that the event on the left-hand side is equivalent to

$$
\left|\frac{\mathrm{F}(n)}{n^{\frac{d-p}{d}}}-\frac{\mathbb{E}(\mathrm{F}(n))}{n^{\frac{d-p}{d}}}\right|>O\left(\frac{(\log n)^{\frac{1}{2}+\frac{p}{d}}}{\sqrt{n}}\right),
$$

where $O(1 / \log n)<\varepsilon / 2$ for sufficiently large $n_{1}$ and $n \geq n_{1}$. Let $n_{2}=\max \left\{n_{0}, n_{1}\right\}$. Then

$$
\sum_{n=1}^{\infty} \mathbb{P}\left(\left|\frac{\mathrm{PA}(X)}{n^{\frac{d-p}{d}}}-\gamma_{\mathrm{F}}^{d, p}\right|>\varepsilon\right) \leq n_{2}+\sum_{n=n_{2}+1}^{\infty} n^{-2}=n_{2}+O(1)<\infty .
$$

Although similar in flavor, smoothness in mean does not immediately imply typical smoothness or vice versa: the latter makes only a statement about single points at worstcase positions. The former only makes a statement about adding and removing several points at random positions. However, the proofs of smoothness in mean for MST and PA do not exploit this, and we can adapt them to yield typical smoothness.

Lemma 4.8. PA and MST are typically smooth.

Proof. We first consider PA. Replacing a point $U_{k}$ by some other (worst-case) point $z$ can be modeled by removing $U_{k}$ and adding $z$. We observe that, in the proof of smoothness in mean (Lemma 3.15), we did not exploit that the point added is at a random position, but the proof goes through for any single point at an arbitrary position. Also the other way around, i.e., removing $z$ and replacing it by a random point $U_{k}$, works in the same way. Thus, PA is typically smooth. 
Closely examining Yukich's proof of smoothness in mean for MST [27, Lemma 4.8] yields the same result for MST.

Corollary 4.9. For all $d$ and $p$ with $p \geq 1, \operatorname{MST}(n) / n^{\frac{d-p}{d}}$ and $\mathrm{PA}(n) / n^{\frac{d-p}{d}}$ converge completely to constants $\gamma_{\mathrm{MST}}^{d, p}$ and $\gamma_{\mathrm{PA}}^{d, p}$, respectively.

Proof. Both MST and PA are typically smooth and converge in mean. Thus, the corollary follows from Theorem 4.7 .

\section{AVERAGE-CASE APPROXIMATION RATIO OF THE MST HEURISTIC}

In this section, we show that the average-case approximation ratio of the MST heuristic for power assignments is strictly better than its worst-case ratio of 2 . First, we prove that the average-case bound is strictly (albeit marginally) better than 2 for any combination of $d$ and $p$. Second, we show a simple improved bound for the 1-dimensional case.

\subsection{The General Case}

The idea behind showing that the MST heuristic performs better on average than in the worst case is as follows: the weight of the PA graph obtained from the MST heuristic can not only be upper-bounded by twice the weight of an MST, but it is in fact easy to prove that it can be upper-bounded by twice the weight of the heavier half of the edges of the MST [5]. Thus, we only have to show that the lighter half of the edges of the MST contributes $\Omega\left(n^{\frac{d-p}{d}}\right)$ to the value of the MST in expectation.

For simplicity, we assume that the number $n=2 m+1$ of points is odd. The case of even $n$ is similar but slightly more technical. We draw points $X=\left\{U_{1}, \ldots, U_{n}\right\}$ as described above. Let $\mathrm{PT}(X)$ denote the power required in the power assignment obtained from the MST. Furthermore, let $\mathrm{H}$ denote the $m$ heaviest edges of the MST, and let $\mathrm{L}$ denote the $m$ lightest edges of the MST. We omit the parameter $X$ since it is clear from the context. Then, by the reasoning above, we have

$$
\mathrm{H}+\mathrm{L}=\mathrm{MST} \leq \mathrm{PA} \leq \mathrm{PT} \leq 2 \mathrm{H}=2 \mathrm{MST}-2 \mathrm{~L} \leq 2 \mathrm{MST} .
$$

For distances raised to the power $p$, the expected value of MST is $\left(\gamma_{\mathrm{MST}}^{d, p} \pm o(1)\right) \cdot n^{\frac{d-p}{d}}$. If we can prove that the lightest $m$ edges of the MST are of weight $\Omega\left(n^{\frac{d-p}{d}}\right)$, then it follows that the MST power assignment is strictly less than twice the optimal power assignment. $L$ is lower-bounded by the weight of the lightest $m$ edges of the whole graph without any further constraints. Let $\mathrm{A}=\mathrm{A}(X)$ denote the weight of these $m$ lightest edges of the whole graph. Note that both $\mathrm{L}$ and $\mathrm{A}$ take edge lengths to the power $p$, and we have $\mathrm{A} \leq \mathrm{L}$.

Let $c$ be a small constant to be specified later on. Let $v_{d, r}=\frac{\pi^{d / 2} r^{d}}{\Gamma\left(\frac{n}{2}+1\right)}$ be the volume of a $d$ dimensional ball of radius $r$. For compactness, we abbreviate $c_{d}=\frac{\pi^{d / 2}}{\Gamma\left(\frac{n}{2}+1\right)}$, thus $v_{d, r}=c_{d} r^{d}$. Note that all $c_{d}$ 's are constants since $d$ is constant.

The probability $P_{k}$ that a fixed vertex $v$ has at least $k$ other vertices within a distance of at most $r=\ell \cdot \sqrt[d]{1 / n}$ for some constant $\ell>0$ is bounded from above by

$$
P_{k} \leq\left(\begin{array}{c}
n-1 \\
k
\end{array}\right) \cdot v_{d, r}^{k} \leq \frac{n^{k}\left(c_{d} r^{d}\right)^{k}}{k !}=\frac{n^{k}\left(c_{d} \ell^{d} n^{-1}\right)^{k}}{k !}=\frac{\tilde{c}^{k}}{k !}
$$


for another constant $\tilde{c}=\ell^{d} c_{d}$. This follows from independence and a union bound. The expected number of edges of a specific vertex that have a length of at most $r$ is thus bounded from above by

$$
\sum_{k=1}^{n-1} P_{k} \leq \sum_{k=1}^{n-1} \frac{\tilde{c}^{k}}{k !} \leq \sum_{k=1}^{\infty} \frac{\tilde{c}^{k}}{k !}=e^{\tilde{c}}-1 .
$$

By choosing $\ell$ appropriately small, we can achieve that $\tilde{c} \leq 1 / 3$. This yields $e^{\tilde{c}}-1<1 / 2$. By linearity of expectation, the total number of edges of length at most $r$ in the whole graph is bounded from above by $m / 2$. Thus, at least $m / 2$ of the lightest $m$ edges of the whole graph have a length of at least $r$. Hence, the expected value of $A$ is bounded from below by

$$
\mathbb{E}(A) \geq \frac{m}{2} \cdot r^{p}=\frac{m}{2} \cdot \ell^{p} n^{-\frac{p}{d}} \leq \frac{\ell^{p}}{4} \cdot n^{\frac{d-p}{d}}=C \cdot n^{\frac{d-p}{d}}
$$

for some constant $C>0$ that depends only on $d$ and $p$. Then the expected value of PT is bounded from above by

$$
\left(2 \gamma_{\mathrm{MST}}^{d, p}-2 C+o(1)\right) \cdot n^{\frac{d-p}{d}}
$$

by (5). From this and the convergence of PA, we can conclude the following theorem.

Theorem 5.1. For any $d \geq 1$ and any $p \geq 1$, we have

$$
\gamma_{\mathrm{MST}}^{d, p} \leq \gamma_{\mathrm{PA}}^{d, p} \leq 2\left(\gamma_{\mathrm{MST}}^{d, p}-C\right)<2 \gamma_{\mathrm{MST}}^{d, p}
$$

for some constant $C>0$ that depends only on $d$ and $p$.

By exploiting that in particular PA converges completely, we can obtain a bound on the expected approximation ratio from the above result.

Corollary 5.2. For any $d \geq 1$ and $p \geq 1$ and sufficiently large $n$, the expected approximation ratio of the MST heuristic for power assignments is bounded from above by a constant strictly smaller than 2.

Proof. The expected approximation ratio is $\mathbb{E}(\mathrm{PT}(n) / \mathrm{PA}(n))=\mathbb{E}\left(\frac{\mathrm{PT}(n) / n \frac{d-p}{d}}{\mathrm{PA}(n) / n \frac{d-p}{d}}\right)$. We know that $\mathrm{PA}(n) / n^{\frac{d-p}{d}}$ converges completely to $\gamma_{\mathrm{PA}}^{d, p}$. This implies that the probability that $\mathrm{PA}(n) / n^{\frac{d-p}{d}}$ deviates by more than $\varepsilon>0$ from $\gamma_{\mathrm{PA}}^{d, p}$ is $o(1)$ for any $\varepsilon>0$.

If $\mathrm{PA}(n) / n^{\frac{d-p}{d}} \in\left[\gamma_{\mathrm{PA}}^{d, p}-\varepsilon, \gamma_{\mathrm{PA}}^{d, p}+\varepsilon\right]$, then the expected approximation ratio can be bounded from above by $\frac{2 \gamma_{\mathrm{MSS}}^{d, p}-2 C}{\gamma_{\mathrm{PA}}^{d, p}-\varepsilon}$. This is strictly smaller than 2 for a sufficiently small $\varepsilon>0$.

Otherwise, we bound the expected approximation ratio by the worst-case ratio of 2 , which contributes only $o(1)$ to its expected value.

Remark 5.3. Complete convergence of the functional PT as well as smoothness and closeness in mean has been shown for the specific case $p=d$ [5]. We believe that PT converges completely for all $p$ and $d$. Since then $\gamma_{\mathrm{PT}}^{d, p} \leq 2 \gamma_{\mathrm{MST}}^{d, p}-2 C<2 \gamma_{\mathrm{MST}}^{d, p}$, we would obtain a simpler proof of Corollary 5.2. 


\subsection{Improved Bound for the One-Dimensional Case}

The case $d=1$ is much simpler than the general case, because the MST is just a Hamiltonian path starting at the left-most and ending at the right-most point. Furthermore, we also know precisely what the MST heuristic does: assume that a point $x_{i}$ lies between $x_{i-1}$ and $x_{i+1}$. The MST heuristic assigns power $\mathrm{PA}\left(x_{i}\right)=\max \left\{\left|x_{i}-x_{i-1}\right|,\left|x_{i}-x_{i+1}\right|\right\}^{p}$ to $x_{i}$. The example that proves that the MST heuristic is no better than a worst-case 2-approximation shows that it is bad if $x_{i}$ is very close to either side and good if $x_{i}$ is approximately in the middle between $x_{i-1}$ and $x_{i+1}$. Too keep the analysis simple, we restrict ourselves to $p \in \mathbb{N}$.

In order to analyze the average-case approximation ratio, we exploit the following trick [12, Chapter 9]: Let $y_{1}, \ldots, y_{n+1}$ be drawn independently according to an exponential distribution with parameter 1 . Let $s_{i}=\sum_{j=1}^{i} y_{i}$, and let $z_{i}=\frac{s_{i}}{s_{n+1}}$. Then $\left\{z_{1}, \ldots, z_{n}\right\}$ is a set of points drawn independently from $[0,1]$ according to the uniform distribution.

Now we observe that the

$$
\mathrm{MST}=\sum_{i=1}^{n-1}\left(z_{i+1}-z_{i}\right)^{p}=\frac{\sum_{i=2}^{n} y_{i}^{p}}{s_{n+1}^{p}} .
$$

The total power for the power assignment obtained from this tree is

$$
\begin{aligned}
\mathrm{PT} & =\left(z_{2}-z_{1}\right)^{p}+\sum_{i=2}^{n-2} \max \left\{z_{i+1}-z_{i}, z_{i}-z_{i-1}\right\}^{p}+\left(z_{n}-z_{n-1}\right)^{p} \\
& =\frac{y_{2}^{p}+\sum_{i=2}^{n-1} \max \left\{y_{i+1}, y_{i}\right\}^{p}+y_{n}^{p}}{s_{n+1}^{p}}
\end{aligned}
$$

Thus,

$$
\frac{\mathrm{PT}}{\mathrm{MST}}=\frac{\overbrace{y_{2}^{p}+\sum_{i=2}^{n-1} \max \left\{y_{i+1}, y_{i}\right\}^{p}+y_{n}^{p}}^{=N}}{\underbrace{\sum_{i=2}^{n} y_{i}^{p}}_{=D}} .
$$

The expected value of the denominator is $\mathbb{E}(D)=(n-1) \cdot p !$. The expected value of the numerator is $\mathbb{E}(N)=\left(2+(n-2) \cdot\left(2-2^{-p}\right)\right) \cdot p$ !, which can be computed by exploiting that the maximum of two exponentially random variables $A$ and $B$ with the same parameter is distributed as $A+\frac{1}{2} B$. This implies that the expected value of $\max \{A, B\}^{p}$ is given by

$$
\begin{aligned}
\mathbb{E}\left(\max \{A, B\}^{p}\right) & =\mathbb{E}\left(\left(A+\frac{1}{2} \cdot B\right)^{p}\right)=\mathbb{E}\left(\sum_{i=0}^{p}\left(\begin{array}{c}
p \\
i
\end{array}\right) \cdot A^{p-i}\left(\frac{1}{2} \cdot B\right)^{i}\right) \\
& =\mathbb{E}\left(\sum_{i=0}^{p}\left(\begin{array}{c}
p \\
i
\end{array}\right) \cdot(p-i) ! \cdot 2^{-i} \cdot i !\right)=p ! \cdot \sum_{i=0}^{p} 2^{-i}=2-2^{-p} .
\end{aligned}
$$

The second equality is the binomial theorem, the third equality holds since $A$ and $B$ are independent and the $k$-th moment of an exponentially distributed random variable is $k !$. 
Note that the ratio of the expected values approaches $2-2^{-p}$ for large $n$.

What remains to be proved is that the probability that the numerator or denominator deviate significantly from the expected values is $o(1)$. This yields then an expected approximation ratio of at most $2-2^{-p}+o(1)$.

The denominator $D$ consists of $n-1$ independent terms of variance $(2 p) !-(p !)^{2}=\Theta(1)$. Thus, the variance of the denominator is $\Theta(n)$. By Chebychev's inequality, we have

$$
\mathbb{P}(|D-\mathbb{E}(D)| \geq t) \leq \frac{\Theta(n)}{t^{2}} .
$$

Plugging in $t=n^{3 / 4}$ yields that $D$ is within a factor of $1 \pm o(1)$ of its expected value is $o(1)$. The numerator can be analyzed similarly. To get rid of the dependencies, we split the sum as follows. Let $n$ be even. Then

$$
\begin{aligned}
& N_{1}=y_{2}^{p}+\sum_{i=2,4, \ldots, n-2} \max \left\{y_{i+1}, y_{i}\right\}^{p}+y_{n}^{p} \text { and } \\
& N_{2}=\sum_{i=3,5, \ldots, n-1} \max \left\{y_{i+1}, y_{i}\right\}^{p} .
\end{aligned}
$$

(For odd $n$, we add the term $y_{n}^{p}$ to $N_{2}$. We skip this case because it is almost identical to the case of even $n$.) Both $N_{1}$ and $N_{2}$ consist of $\Theta(n)$ independent random variables of constant variance and we have $N=N_{1}+N_{2}$. Concentration for both and, thus, for $N$ can be shown in the same way as for $D$. Therefore, we obtain the following results.

Theorem 5.4. For all $p \geq 1$, we have $\gamma_{\mathrm{MST}}^{1, p} \leq \gamma_{\mathrm{PA}}^{1, p} \leq\left(2-2^{-p}\right) \cdot \gamma_{\mathrm{MST}}^{1, p}$.

Proof. The first inequality is immediate. The second inequality follows from $\mathrm{PA}(X) \leq$ $\mathrm{PT}(X)$ for all $X$ and the reasoning above.

Corollary 5.5. The expected approximation ratio of the MST heuristic is at most $2-2^{-p}+$ $o(1)$.

Proof. The expected values of PT and MST differ by a factor of $2-2^{-p}+o(1)$. The approximation ratio is given by (6). The probability that numerator or denominator differ by more than a factor of $1 \pm o(1)$ from their expected value is $o(1)$. And if indeed numerator or denominator differ significantly from their expected value, we apply the worst-case approximation ratio of 2 , which adds only $o(1)$ to the expected approximation ratio.

While this does not follow from our results, we conjecture that the MST heuristic does not yield asymptotically optimal power assignments. In fact, we conjecture that $\mathrm{PT}(n) / \mathrm{PA}(n)$ converges to a constant strictly greater than 1 .

\section{CONCLUSIONS AND OPEN PROBLEMS}

We have proved complete convergence of Euclidean functionals that are typically smooth (Definition 4.4) for the case that the distance-power gradient $p$ is larger than the dimension $d$. The case $p>d$ appears naturally in the case of transmission questions for wireless networks. 
As examples, we have obtained complete convergence for the MST (minimum-spanning tree) and the PA (power assignment) functional. To prove this, we have used a recent concentration of measure result by Warnke [26]. His concentration inequality might be of independent interest to the algorithms community. As a technical challenge, we have had to deal with the fact that the degree of an optimal power assignment graph can be unbounded.

To conclude this paper, let us mention some problems for further research:

1. Is it possible to prove complete convergence of other functionals for $p \geq d$ ? The most prominent one would be the traveling salesman problem (TSP). However, we are not aware that the TSP is smooth in mean for $p \geq d$, which would be a necessary property to apply our method.

2. Concerning the average-case approximation ratio of the MST heuristic, we only proved that the approximation ratio is smaller than 2. Only for the case $d=1$, we provided an explicit upper bound for the approximation ratio. Is it possible to provide an improved approximation ratio as a function of $d$ and $p$ for general $d$ ?

3. Can Rhee's isoperimetric inequality [22] be adapted to work for $p \geq d$ ? Rhee's inequality can be used to obtain convergence for the case that the points are not identically distributed, and has for instance been used for a smoothed analysis of Euclidean functionals [2]. (Smoothed analysis has been introduced by Spielman and Teng to explain the performance of the simplex method [23]. We refer to two surveys for an overview $[16,24]$.)

4. Can our findings about power assignments be generalized to other problems in wireless communication, such as the $k$-station network coverage problem of Funke, Laue, Lotker, and Naujoks [6], where transmit powers are assigned to at most $k$ stations such that $X$ can be reached from at least one sender, or power assignments in the SINR model $[8,10]$ ? Interestingly, in the SINR model the MST turns out to be a good solution to schedule all links within a short time $[9,11]$.

More general, can this framework also be exploited to analyze other approximation algorithms for geometric optimization problems? As far as we are aware, besides partitioning heuristics [2,27], the only other algorithm analyzed within this framework is Christofides' algorithm for the TSP [3].

\section{ACKNOWLEDGMENTS}

We thank Samuel Kutin, Lutz Warnke, and Joseph Yukich for fruitful discussions.

\section{REFERENCES}

[1] E. Althaus, G. Calinescu, I. I. Mandoiu, S. K. Prasad, N. Tchervenski, and A. Zelikovsky, Power efficient range assignment for symmetric connectivity in static ad hoc wireless networks, Wireless Networks 12 (2006), 287-299.

[2] M. Bläser, B. Manthey, and B. V. Raghavendra Rao, Smoothed analysis of partitioning algorithms for Euclidean functionals, Algorithmica 66 (2013), 397-418.

[3] M. Bläser, K. Panagiotou, and B. V. Raghavendra Rao, A probabilistic analysis of Christofides' algorithm, In F. V. Fomin and P. Kaski, editors, Proceedings of the 13th Scandinavian Workshop on Algorithm Theory (SWAT), Volume 7357 of Lecture Notes in Computer Science, Springer, Helsinki, Finland, 2012, pp 225-236. 
[4] A. E. F. Clementi, P. Penna, and R. Silvestri, On the power assignment problem in radio networks, Mobile Networks Appl 9 (2004), 125-140.

[5] M. de Graaf, R. J. Boucherie, J. L. Hurink, and J.-K. van Ommeren, An average case analysis of the minimum spanning tree heuristic for the range assignment problem, Memorandum 11259 (revised version), Department of Applied Mathematics, University of Twente 2013.

[6] S. Funke, S. Laue, Z. Lotker, and Rouven Naujoks, Power assignment problems in wireless communication: Covering points by disks, reaching few receivers quickly, and energy-efficient travelling salesman tours, Ad Hoc Networks 9 (2011) 1028-1035.

[7] P. Gupta and P. R. Kumar, Critical power for asymptotic connectivity in wireless networks, In W. M. McEneaney, G. George Yin, and Q. Zhang, editors, Stochastic analysis, control, optimization and applications: A volume in honor of W.H. Fleming, Systems \& control: Foundations \& applications, Springer, 1999, pp. 547-566.

[8] M. M. Halldórsson, S. Holzer, P. Mitra, and R. Wattenhofer, The power of non-uniform wireless power, Proceedings of the 24th Annual ACM-SIAM Symposium on Discrete Algorithms (SODA), SIAM, New Orleans, LA, USA, 2013, pp. 1595-1606.

[9] M. M. Halldórsson and P. Mitra, Wireless connectivity and capacity, In Y. Rabani, editor, Proceedings of the 23rd Annual ACM-SIAM Symposium on Discrete Algorithms (SODA), SIAM, Kyoto, Japan, 2012, pp, 516-526.

[10] T. Kesselheim, A constant-factor approximation for wireless capacity maximization with power control in the SINR model, Proceedings of the 22nd Annual ACM-SIAM Symposium on Discrete Algorithms (SODA), SIAM, San Francisco, CA, USA, 2011, pp. 1549-1559.

[11] M. Khan, G. Pandurangan, G. Pei, and A. K. S. Vullikanti, Brief announcement: A fast distributed approximation algorithm for minimum spanning trees in the SINR model, In M. K. Aguilera, editor, Proceedings of the 26th International Symposium on Distributed Computing (DISC), volume 7611 of Lecture Notes in Computer Science, Springer, Salvador, Brazil, 2012, pp. 409-410.

[12] J. F. C. Kingman, Poisson processes, Volume 3 of Oxford studies in probability, Oxford University Press, 1993.

[13] L. M. Kirousis, E. Kranakis, D. Krizanc, and A. Pelc, Power consumption in packet radio networks, Theore Comput Sci 243 (2000), 289-305.

[14] G. Kozma, Z. Lotker, and G. Stupp, On the connectivity threshold for general uniform metric spaces, Inf Process Lett 110 (2010), 356-359.

[15] E. L. Lloyd, R. Liu, M. V. Marathe, R. Ramanathan, and S. S. Ravi, Algorithmic aspects of topology control problems for ad hoc networks, Mobile Networks Appl 10 (2005) 19-34.

[16] B. Manthey and H. Röglin, Smoothed analysis: Analysis of algorithms beyond worst case, Inf Tech 53 (2011), 280-286.

[17] M. Mitzenmacher and E. Upfal, Probability and computing: Randomized algorithms and probabilistic analysis, Cambridge University Press, 2005.

[18] K. Pahlavan and A. H. Levesque, Wireless information networks, Wiley, 1995.

[19] M. D. Penrose, The longest edge of the random minimal spanning tree, Ann Appl Probab 7 (1997), 340-361.

[20] M. D. Penrose, A strong law for the longest edge of the minimal spanning tree, Ann Probab 27 (1999), 246-260.

[21] T. S. Rappaport, Wireless communication, Prentice Hall, 2002.

[22] W. T. Rhee, A matching problem and subadditive Euclidean functionals, Ann Appl Probab 3 (1993), 794-801.

[23] D. A. Spielman and S.-H. Teng, Smoothed analysis of algorithms: Why the simplex algorithm usually takes polynomial time, J ACM 51 (2004), 385-463. 
[24] D. A. Spielman and S.-H. Teng, Smoothed analysis: An attempt to explain the behavior of algorithms in practice, Commun ACM 52 (2009), 76-84.

[25] J. Michael Steele, Probabilistic and worst case analyses of classical problems of combinatorial optimization in Eclidean space, Math Oper Res 15 (1990), 749-770.

[26] L. Warnke, On the method of typical bounded differencesm, Combin, Probab Comput 25 (2016), 269-299.

[27] J. E. Yukich, Probability theory of classical euclidean optimization problems, Volume 1675 of lecture notes in mathematics, Springer, 1998. 